Изв. Крымск. Астрофиз. Обсерв. 116, № 2, 34-40 (2020) doi:10.31059/izcrao-vol116-iss2-pp34-40

\author{
УДК 524.3-355
}

\title{
Исследование многолетней спектральной переменности Ае звезды Хербига HD 36112
}

\author{
М.И. Фурсяк, О.В. Козлова \\ ФГБУН “Крымская астрофизическая обсерватория РАН”, Научный, Крым, 298409 \\ mbjhht@yandex.ru
}

Поступила в редакцию 7 октября 2020 г.

\begin{abstract}
Аннотация. Представлены результаты многолетних высокодисперсионных спектральных наблюдений $(\mathrm{R}=20000)$ Ае звезды Хербига HD 36112 в области эмиссионных линий $\mathrm{H}_{\alpha}$ и резонансного дублета NaI D. Показано, что параметры эмиссионной линии $\mathrm{H}_{\alpha}$ демонстрируют сложную переменность на нескольких временных шкалах: 1) переменность от ночи к ночи, обусловленную неоднородной структурой околозвездной газовой оболочки; 2) переменность на шкале времени около 1200 суток, характеризующуюся изменением эквивалентной ширины, интенсивности, а также других параметров эмиссии; 3) переменность на шкале времени более 4000 суток, наблюдаемую как многолетний тренд в изменении параметров эмиссионной линии $\mathrm{H}_{\alpha}$.

Мы связываем полученные результаты с изменением физических и кинематических условий во внутренних областях аккреционного диска и ветра. Наиболее вероятным механизмом таких изменений является активно идущий в околозвездном диске звезды процесс планетообразования.
\end{abstract}

Ключевые слова: Ае звезды Хербига, спектральная переменность, планетообразование, ветер, околозвездные диски

\section{1 Введение}

Звезда HD 36112 (MWC 758) относится к группе молодых горячих звезд промежуточных масс или Ае/Ве звезд Хербига. Они окружены остатками родительских облаков, представляющих собой протяженные газопылевые диски, в которых идут активные процессы планетообразования. Хорошо известно, что в их внутренних областях происходит аккреция околозвездного (CS) вещества на звезду, тогда как в более высоких широтах наблюдаются процессы истечения газа - ветер (см., напр., Alecian et al., 2013). Поэтому Ае/Ве звезды Хербига характеризуются присутствием в их спектрах CS эмиссионных линий, формирующихся в газовой оболочке, а также ИК-избытками, связанными с излучением околозвездной пыли. Это верно и в отношении HD 36112.

Хотя звезда находится в известной области звездообразования Телец - Возничий, она была отнесена к молодым звездам только в 1990-е годы, после обнаружения у нее ИК-избытков (The et al., 1994). По данным Palla and Stahler (1993), масса звезды составляет 1.8 масс Солнца, а возраст около 5-10 миллионов лет, что близко к оценкам возраста других Ае/Ве звезд Хербига. Спектральный класс звезды A8Ve, блеск в полосе $\mathrm{V}$ составляет $8^{m} .3$, а период осевого вращения - около $1.8^{d}$ (Beskrovnaya et al., 1999).

Фотометрические исследования HD 36112 довольно разрозненны. Это обусловлено ее слабой фотометрической активностью (амплитуда переменности не превышает нескольких десятых зв. величины по данным Beskrovnaya et al., 1999) и связано с ориентацией звезды по отношению к наблюдателю. Так, по данным ИК-интерферометрии Eisner et al. (2004) и Isella et al. (2008), угол наклона оси вращения HD 36112 к лучу зрения составляет около 30-40. Это означает, что звезда ориентирована под промежуточным углом, при котором на луче зрения оказывается область ветра (Grinin, Rostopchina, 1996).

Подробный анализ быстрой спектральной переменности HD 36112 выполнен в работах Pogodin (1995) и Beskrovnaya et al. (1999). Результаты этих исследований показали, что CS эмиссионные 
линии в спектре имеют профиль типа P Cyg, характерный для истекающего газа. Сильную переменность параметров эмиссионных линий от ночи к ночи авторы связывают с присутствием азимутальных неоднородностей в структуре ветра. K сожалению, у нас нет данных о спектральной переменности звезды на других временных шкалах.

Недавние исследования структуры диска HD 36112 в миллиметровом и ближнем ИК-диапазонах (Boehler et al., 2018; Benisty et al., 2015) показали, что в околозвездном диске HD 36112 активно формируется молодая планетная система. Поскольку данные моделирования показывают, что процесс планетообразования (наряду с двойственностью) способен не просто изменять структуру дисков, но и вызывать изменение темпа аккреции на шкале времени, равной орбитальному периоду (см. Демидова, 2016, 2009), мы попытались выявить наблюдаемые признаки таких изменений, опираясь на данные многолетнего спектрального мониторинга HD 36112, проводимого в Крымской астрофизической обсерватории.

\section{2 Наблюдения}

Спектры HD 36112 высокого разрешения $(\mathrm{R}=20000)$ были получены в период с 2009 по 2018 гг. в КрАО на 2.6-м телескопе (ЗТШ). До 2013 года спектры получались с помощью куде-спектрографа АСП-14 и ПЗС-камеры, с конца 2013 года наблюдения велись на эшельном спектрографе ЭСПЛ, установленном в фокусе куде. Всего было получено 53 спектра в области эмиссионной линии $\mathrm{H}_{\alpha}$ и 13 спектров в области линий резонансного дублета натрия NaI D.

Обработка спектров выполнялась с помощью пакета программ SPE, разработанных С.Г. Сергеевым и используемых в КрАО.

При определении параметров эмиссионной линии $\mathrm{H}_{\alpha}$ и резонансного дублета натрия $\mathrm{NaI} \mathrm{D}$ мы вычитали из наблюдаемого спектра синтетический (такая практика является общепринятой при анализе эмиссионных профилей). Для этого были рассчитаны спектры, соответствующие модели $T_{\text {eff }}=7750 \mathrm{~K}, \lg (g)=4$ и $v \sin i=55 \mathrm{~km} / \mathrm{c}$ (Beskrovnaya et al., 1999). Ошибки определения $E W\left(\mathrm{H}_{\alpha}\right)$ не превышают $0.5 \AA$.

\section{3 Результаты наблюдений}

\section{1 Линия $\mathrm{H}_{\alpha}$}

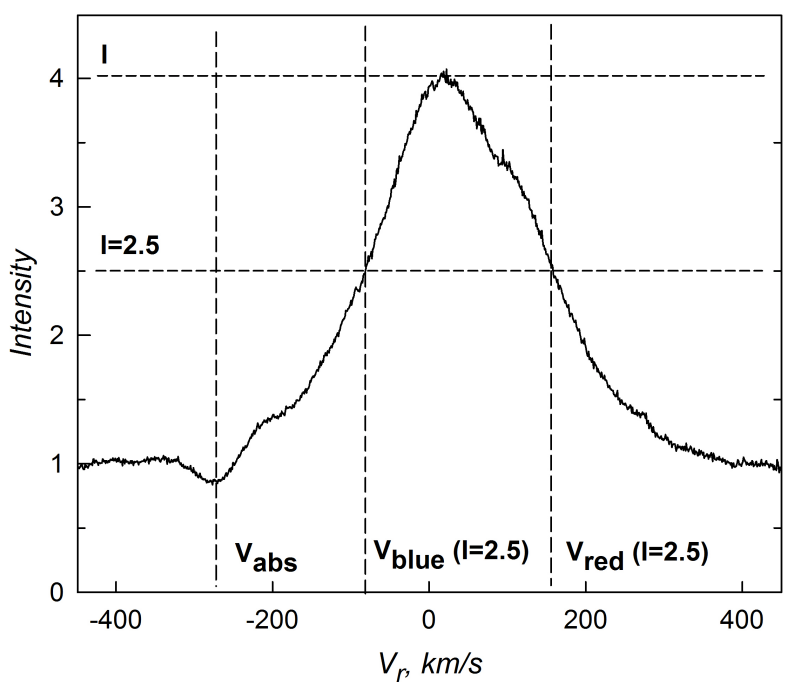

Рис. 1. Пример определения параметров эмиссионной линии $\mathrm{H}_{\alpha}$ профиля для уровней интенсивности $I=2.5$. По двум последним параметрам мы оценили также ширину эмиссии $W_{\mathrm{em}}(I=2.5)$ и значения бисектора $V_{\mathrm{bis}}(I=2.5)$. Изменение этих величин со временем показано на рис. 2 и рис. 3.

Пример спектра HD 36112 в области эмиссионной линии $\mathrm{H}_{\alpha}$ показан на рис. 1. Видно, что эта линия представляет собой широкую эмиссию, в синем крыле которой могут наблюдаться переменные абсорбционные компоненты, формирующиеся в ветре (профиль типа Р Cyg). В отдельные ночи вместо одной абсорбции могут наблюдаться две абсорбционные компоненты. Это позволило нам выделить низкоскоростную (лучевые скорости не превышают -200 км/с) и высокоскоростную ветровые компоненты. Однако нельзя исключать, что число этих компонент еще больше.

Для анализа многолетней переменности эмиссии мы выделили несколько параметров (см. рис. 1). Среди них эквивалентная ширина эмиссии $E W\left(\mathrm{H}_{\alpha}\right)$ и ее максимальная интенсивность $I$, лучевые скорости смещенных в синюю сторону абсорбционных компонент $V_{\text {abs }}$ и их интенсивности $I_{\mathrm{abs}}$, а также лучевые скорости синей $V_{\text {blue }}$ и красной $V_{\text {red }}$ границ эмиссионного 
На рисунках хорошо заметны изменения эмиссионных параметров от ночи к ночи. Такой тип переменности подробно описан в работе (Beskrovnaya et al., 1999) и связан с неоднородной структурой околозвездной газовой оболочки. Кроме того, можно заметить в изменении $I$ и $E W\left(\mathrm{H}_{\alpha}\right)$ несколько минимумов значений, наблюдавшихся в даты J.D. 2455100, 2456300 и 2458000 (см. рис. 2). Наоборот, вблизи дат J.D. 2456000, 2457200 и 2458300 наблюдается рост этих величин. Интересно, что одновременно с этим происходит резкое увеличение скоростей смещенных в синюю сторону абсорбционных компонент $V_{\text {abs }}$ и рост их глубин (см. переменность $\left.I_{\text {abs }}\right)$.

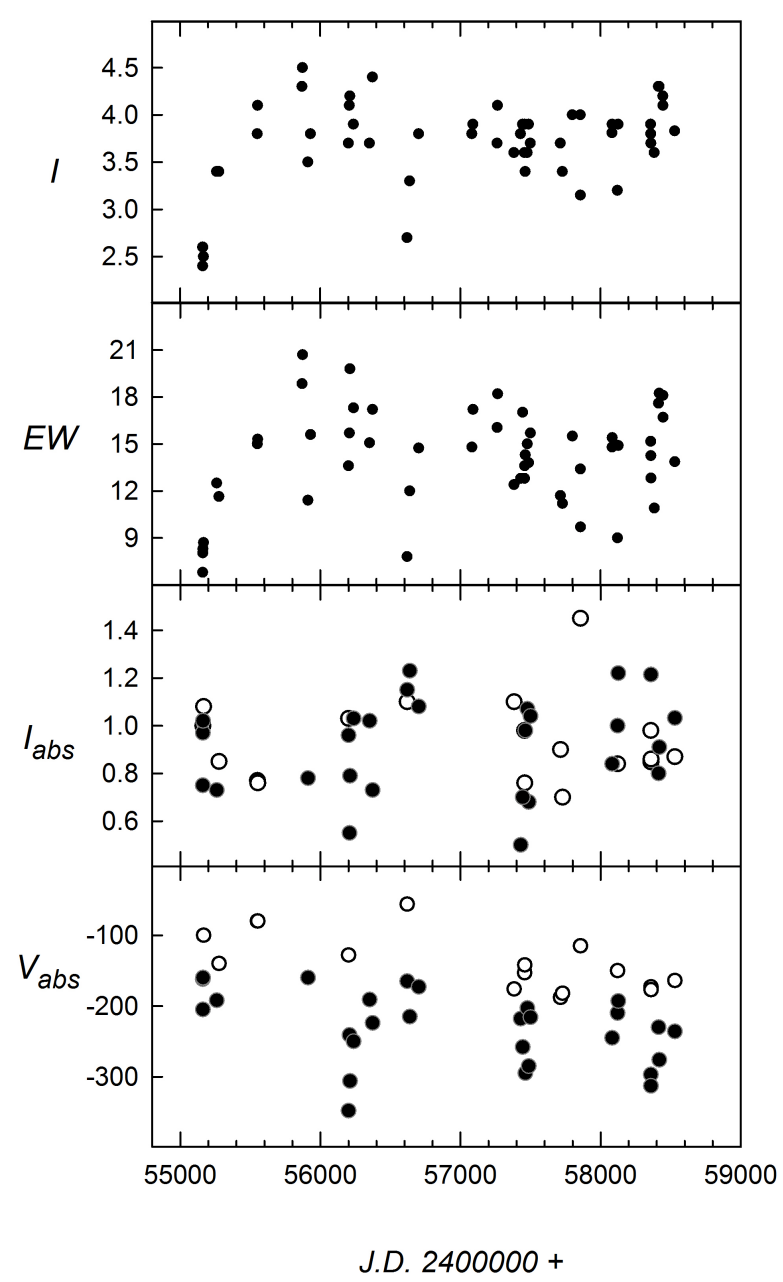

Рис. 2. Многолетняя переменность параметров эмиссионной линии $\mathrm{H}_{\alpha}$ : эквивалентной ширины $E W$, интенсивности $I$, а также лучевых скоростей ветровых компонент $V_{\text {abs }}$ и их интенсивностей $I_{\text {abs }}$ (черными кружками показаны высокоскоростные компоненты ветра, пустыми кружками - низкоскоростные)

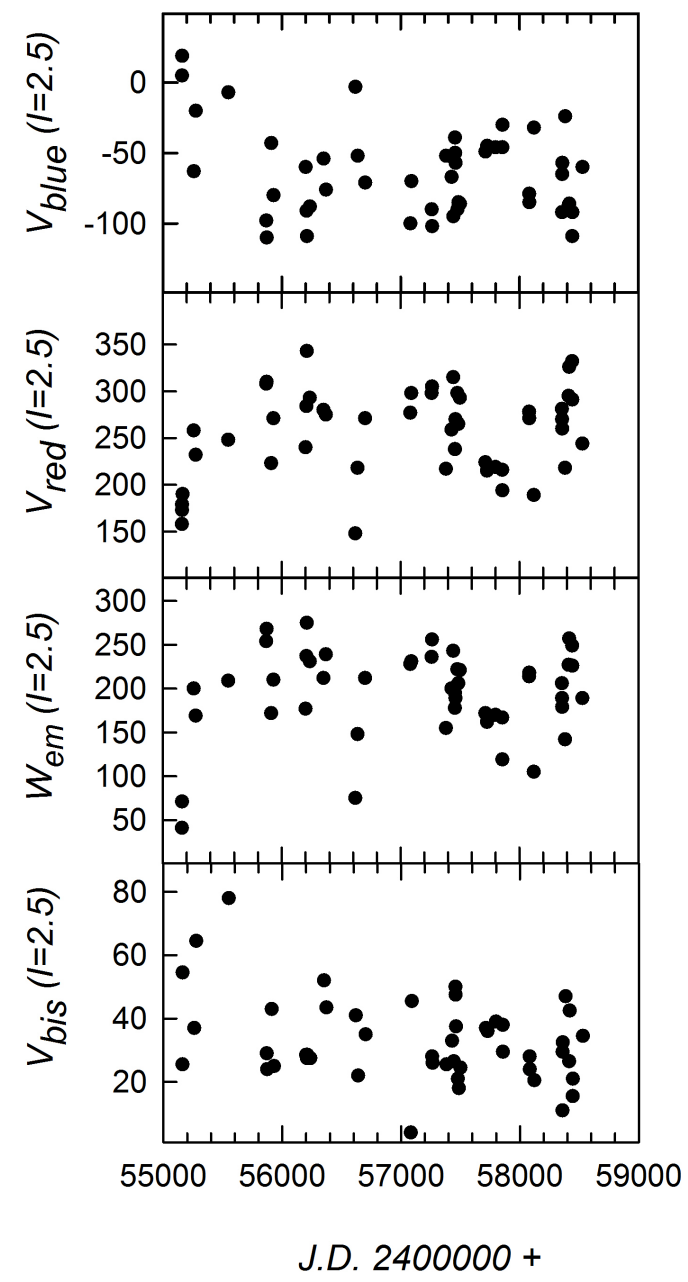

Рис. 3. Многолетняя переменность параметров эмиссионной линии $\mathrm{H}_{\alpha}$ : лучевых скоростей синей $V_{\text {blue }}$ и красной $V_{\text {red }}$ границ эмиссионного профиля, ширины эмиссии $W_{\text {em }}$ и положения бисектора $V_{\text {bis }}$ для уровня интенсивности $I=2.5$

Анализ рис. 3 приводит нас к тем же выводам: в даты наибольших значений $E W\left(\mathrm{H}_{\alpha}\right)$ и $\mathrm{I}$ отмечается увеличение лучевых скоростей синей $V_{\text {blue }}(I=2.5)$ и красной $V_{\text {red }}(I=2.5)$ границ эмиссии, т. е. на шкале времени 1200 суток изменяется ширина эмиссии (см. рис. 3). Интересно, что в изменении величины бисектора $V_{\mathrm{bis}}(I=2.5)$ мы не видим какой-либо значимой переменности на этой шкале. Можно также предположить существование еще одной шкалы переменности. Она выглядит как многолетний тренд в изменении $E W\left(\mathrm{H}_{\alpha}\right)$ и $V_{\mathrm{abs}}$, охватывающий весь период наблюдений (более 4000 суток). 
Таким образом, полученные результаты показывают существование некоторого характерного времени (около $1200^{d}$ ), на котором в околозвездной газовой оболочке HD 36112 изменяются физические и кинематические характеристики истекающего CS вещества. По-видимому, мы можем говорить о циклах активности, во время которых процесс истечения газа у звезды (ветер) то усиливается, то ослабевает.

\section{2 Линии резонансного дублета натрия $\mathrm{NaI} \mathrm{D}$}

Эмиссионные линии резонансного дублета натрия, наблюдаемые у Ае/Ве звезд Хербига, более слабые, чем линия $\mathrm{H}_{\alpha}$. Они имеют довольно симметричный профиль, который, в отличие от эмиссии $\mathrm{H}_{\alpha}$, формируется в основном в аккреционном диске, а не в ветре. По этой же причине эмиссионные линии NaI D более узкие, а смещенные в синюю сторону абсорбционные компоненты уже не попадают в область синего эмиссионного крыла, как в линиях водорода. Это хорошо видно на рис. 4, где в качестве примера показан спектр HD 36112 в области NaI D, полученный 30 сентября 2012 г.

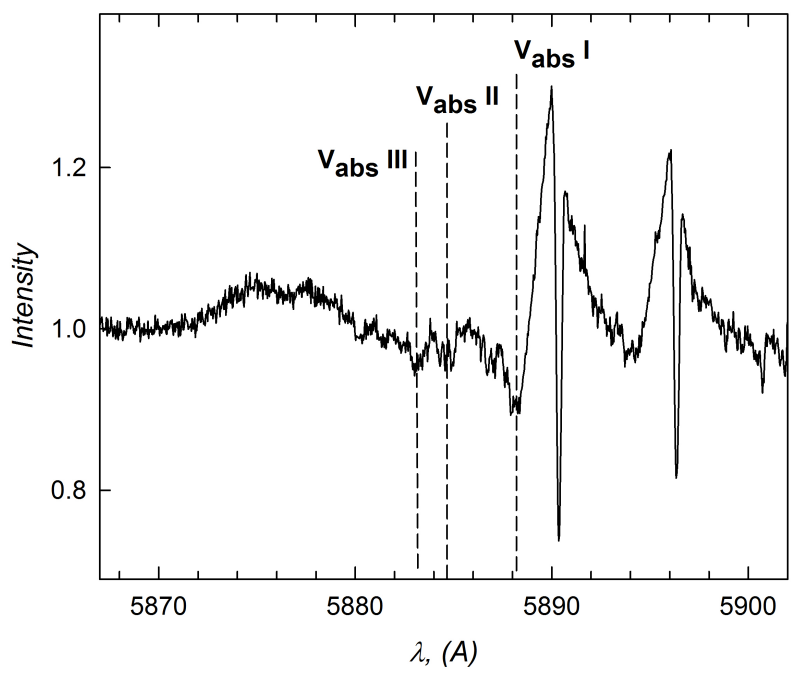

Рис. 4. Пример спектра HD 36112 в области линий резонансного дублета натрия

На широких эмиссионных линиях дублета хорошо заметна узкая межзвездная абсорбция. С синей стороны от эмиссии можно видеть CS абсорбционные компоненты, формирующиеся, как и у линии $\mathrm{H}_{\alpha}$, в ветре. Интересно, что здесь видны уже не две, а три абсорбционные компоненты. Самая низкоскоростная из них имеет скорость $-90 \mathrm{~km} / \mathrm{c}$. В линии $\mathrm{H}_{\alpha}$ линии с такими скоростями практически не видны, т. к. попадают в область сильного градиента интенсивности.

Для анализа переменности линий дублета $\mathrm{NaI}$ D мы определили эквивалентную ширину эмиссии $E W(\mathrm{NaI} \mathrm{D})$ и лучевые скорости ветровых компонент $V_{\mathrm{abs}}(\mathrm{NaI}) . \mathrm{K}$ сожалению, спектров в этой области получено слишком мало, поэтому мы ограничились только сравнением переменности эквивалентных ширин и лучевых скоростей с изменением аналогичных параметров у эмиссионной линии $\mathrm{H}_{\alpha}$. Его результаты показаны на рис. 5 и рис. 6.

Как видно из рисунков, обнаруживается связь между переменностью линий $\mathrm{NaI} \mathrm{D}$ и $\mathrm{H}_{\alpha}$, но количество спектров в области натрия пока не позволяет уверенно говорить о корреляции между этими линиями. Тем не менее, такая корреляция вполне естественна и ожидаема (Козлова и др., 2007a), т. к. обусловлена тесной связью между процессами аккреции и истечения у молодых звезд.

Подтверждение этой корреляции представляется очень важным, т. к. будет означать, что параметры линий дублета NaI D тоже должны показывать переменность на шкале времени 1200 суток. Выше мы уже отмечали, что присутствие в CS диске формирующейся планеты обуславливает изменение темпа аккреции на звезду на шкале времени, равной орбитальному периоду (Демидова, 2009). Поскольку эмиссионные линии $\mathrm{NaI} \mathrm{D}$ формируются в аккреционном диске, мы получим прямое подтверждение изменения темпа аккреции у HD 36112. 


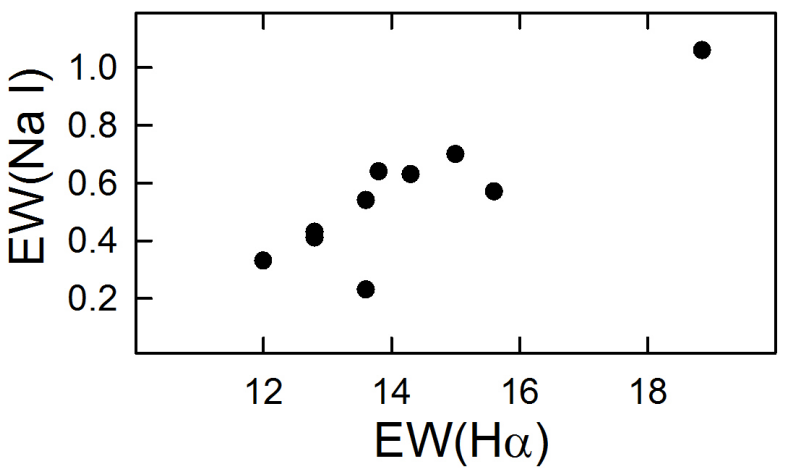

Рис. 5. Связь между изменением эквивалентных ширин $E W(\mathrm{NaI})$ и $E W\left(\mathrm{H}_{\alpha}\right)$

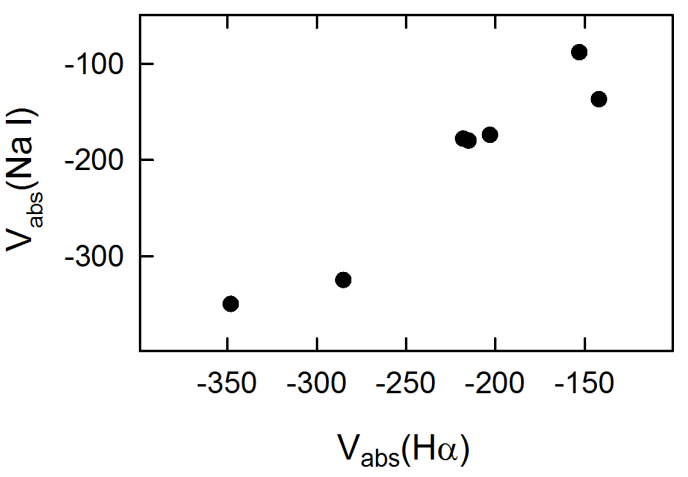

Рис. 6. Связь между изменением лучевых скоростей ветровых компонент $V_{\mathrm{abs}}(\mathrm{NaI})$ и $V_{\mathrm{abs}}\left(\mathrm{H}_{\alpha}\right)$

\section{4 Заключение}

Как уже отмечалось выше, данные многолетнего спектрального мониторинга HD 36112 показали наличие переменности на нескольких временных шкалах. Наибольший интерес представляет шкала переменности около $1200^{d}$, на которой происходят очень сильные изменения параметров истекающего газа (ветра) в линии $\mathrm{H}_{\alpha}$. Они показывают, что ветер то усиливается, то ослабевает, а сама картина оказывается очень похожей на спектральную переменность звезды MWC 480 (Козлова и др., 2007а). Сходство проявляется и в присутствии у MWC 480 более длинной шкалы переменности, наблюдаемой как тренд, охватывающий весь период наблюдений (более 4000 суток). Обе звезды сближает еще один факт: у них не обнаружено никаких признаков двойственности, зато наблюдается активный процесс планетообразования.

Выше уже отмечалось, что фотометрическая переменность HD 36112 из-за промежуточной ориентации ее диска по отношению к наблюдателю не превышает нескольких десятых зв. величины. В то же время существует группа молодых звезд, ориентация которых ближе к положению "с ребра". Это звезды типа UX Ori, показывающие очень сильную фотополяриметрическую активность, обусловленную затмениями, вызываемыми газопылевыми облаками (см., напр., Ростопчина и др., 1999). Многолетний фотополяриметрический мониторинг этих объектов показал, что ослабления блеска у них не случайны, а показывают периодичность на многолетней временной шкале. При этом у одной звезды обнаруживаются несколько таких периодов, самые длинные из которых могут достигать нескольких десятков лет. Их наличие показывает присутствие устойчивых масштабных неоднородностей в структуре CS газопылевых дисков этих звезд. Сегодня нет сомнений в том, что такая структура обусловлена процессом планетообразования.

Нельзя не отметить в этой связи и данные спектрального исследования звезды CQ Tau (Козлова и др., 2007b). Самый короткий из фотометрических периодов звезды составляет около $1000^{d}$ (Шаховской и др., 2005), что близко к спектральной шкале переменности CQ Таu (около $\left.700^{d}\right)$. Более того, данные одновременного спектрального и фотометрического мониторинга этой звезды показали, что именно пылевые облака, вызывающие изменения блеска, втягиваясь во внутреннюю область аккреционного диска, вызывают появление газа с аномальными кинематическими характеристиками и обуславливают необычную спектральную активность звезды. О связи между фотометрической и спектральной переменностью упоминалось и в отношении звезды MWC 480 (Козлова и др., 2007a). В этой связи хочется надеяться, что звезду HD 36112 тоже удастся включить в фотометрический мониторинг.

Все вышесказанное позволяет нам связать результаты многолетнего спектрального мониторинга HD 36112 с формированием массивных планет во внутренней области ее околозвездного диска. Спектральный мониторинг этой звезды будет продолжаться, в том числе и в других спектральных диапазонах.

Авторы благодарны рецензенту за интерес к статье и полезные замечания, которые в значительной степени помогли улучшить данную работу. 


\section{Литература}

Демидова Т.В., 2009. Астрофизика. Т. 52. № 4. С. 623-630. [Demidova T.V., 2009. Astrofizika, vol. 52, no. 4, pp. 623-630. (In Russ.)]

Демидова Т.В., 2016. Астрофизика. Т. 59. № 4. С. 505-518. [Demidova T.V., 2016. Astrofizika, vol. 59, no. 4, pp. 505-518. (In Russ.)]

Козлова О.В., Алексеев И.Ю., Шаховской Д.Н., 2007а. Астрофизика. Т. 50. № 4. С. $565-588$. [Kozlova O.V., Alekseev I.Yu., Shakhovskoi D.N., 2007. Astrofizika, vol. 50, no. 4, pp. 565-588. (In Russ.)]

Козлова О.В., Ростопчина А.Н., Шаховской Д.Н., Алексеев И.Ю., 2007b. Астрофизика. Т. 50. № 1. C. 39-56. [Kozlova O.V., Rostopchina A.N., Shakhovskoi D.N., Alekseev I.Yu., 2007. Astrofizika, vol. 50, no. 1, pp. 39-56. (In Russ.)]

Ростопчина А.Н., Гринин В.П., Шаховской Д.Н., 1999. Письма в Астрон. журн. Т. 25. № 4. С. 291298. [Rostopchina A.N., Grinin V.P., Shakhovskoi D.N., 1999. Pis'ma v Astron. zhurn., vol. 25, no. 4, pp. 291-298. (In Russ.)]

Шаховской Д.Н., Гринин В.П., Ростопчина А.Н., 2005. Астрофизика. Т. 48. № 2. С. $165-174$. [Shakhovskoi D.N., Grinin V.P., Rostopchina A.N., 2005. Astrofizika, vol. 48, no. 2, pp. 165-174. (In Russ.)]

Alecian E., Wade G.A., Catala C., et al., 2013. Mon. Not. Roy. Astron. Soc., vol. 429, pp. 1001-1026.

Benisty M., Juhasz A., Boccaletti A., et al., 2015. Astron. Astrophys., vol. 578, p. L6.

Beskrovnaya N.G., Pogodin M.A., Miroshnichenko A.S., et al., 1999. Astron. Astrophys., vol. 343, pp. 163174.

Boehler Y., Ricci L., Weaver E., et al., 2018. Astrophys. J., vol. 853, pp. 162-176.

Eisner J.A., Lane B.F., Hillenbrand L.A., Akeson R.L., Sargent A.I., 2004. Astrophysp. J., vol. 613, pp. 1049-1071.

Grinin V.P., Rostopchina A.N., 1996. Astron. Rep., vol. 40, iss. 2, pp. 171-178.

Isella A., Tatulli E., Natta A., Testi L., 2008. Astron. Astrophys., vol. 483, p. L13.

Palla F., Stahler W.S., 1993. Astrophys. J., vol. 418, pp. 414-425.

Pogodin M.A., 1995. Astrophys. Space Sci., vol. 224, pp. 539-540.

The P.S., De Winter D., Perez M.R., 1994. Astron. Astropys. Suppl. Ser., vol. 104, pp. 315-339. 


\title{
Investigation of long-term spectral variability of the Ae Herbig star HD 36112
}

\author{
M.I. Fursyak, O.V. Kozlova \\ Crimean Astrophysical Observatory, Nauchny, 298409 \\ mbjhht@yandex.ru
}

\begin{abstract}
We present the results of long-term high-dispersion spectral observations $(\mathrm{R}=20000)$ of Ae Herbig star HD 36112 in the region of the $\mathrm{H}_{\alpha}$ emission line and the NaI D resonance doublet. They show that parameters of the $\mathrm{H}_{\alpha}$ emission line demonstrate the complicated variability on several time scales: 1) the variability from night to night caused by inhomogeneity of the circumstellar envelope; 2) the variability on a time scale of about $1200^{d}$ characterized by variation of the equivalent width, intensity, and other emission parameters; 3 ) the variability on a time scale of more than $4000^{d}$ observed as a long-time trend in variations of parameters of the $\mathrm{H}_{\alpha}$ emission line.

We associate these results with variability of physical and kinematic conditions in the inner regions of the accretion disk and wind. The most probable mechanism of this variability is a process of planet formation in the circumstellar disk.
\end{abstract}

Key words: Ae Herbig stars, spectral variability, planetary formation, wind, circumstellar disks 